\title{
Mangiferin Accelerates Glycolysis and Enhances Mitochondrial Bioenergetics
}

\author{
Zhongbo Liu ${ }^{1}$, Pasha Apontes ${ }^{1}$, Ekaterina V. Fomenko ${ }^{1}$, Nan Chi ${ }^{1}$, Victor L. Schuster ${ }^{1,2}$, \\ Irwin J. Kurland ${ }^{1,3}$, Jeffrey E. Pessin ${ }^{1,4}$ and Yuling Chi ${ }^{1, *}$ \\ 1 Department of Medicine, Albert Einstein College of Medicine, Bronx, NY 10461, USA; \\ liuzb727@gmail.com (Z.L.); p.apontes@gmail.com (P.A.); fomenko875ekaterina@gmail.com (E.V.F.); \\ nanchi2013@yahoo.com (N.C.); victor.schuster@einstein.yu.edu (V.L.S.); \\ Irwin.Kurland@einstein.yu.edu (I.J.K.); Jeffrey.Pessin@einstein.yu.edu (J.E.P.) \\ 2 Department of Physiology and Biophysics, Albert Einstein College of Medicine, Bronx, NY 10461, USA \\ 3 Einstein Stable Isotope and Metabolomics Core, Albert Einstein College of Medicine, Bronx, NY 10461, USA \\ 4 Department of Molecular Pharmacology, Albert Einstein College of Medicine, Bronx, NY 10461, USA \\ * Correspondence: yuling.chi@einstein.yu.edu; Tel.: +1-718-430-8913
}

Received: 1 December 2017; Accepted: 27 December 2017; Published: 9 January 2018

\begin{abstract}
One of the main causes of hyperglycemia is inefficient or impaired glucose utilization by skeletal muscle, which can be exacerbated by chronic high caloric intake. Previously, we identified a natural compound, mangiferin (MGF) that improved glucose utilization in high fat diet (HFD)-induced insulin resistant mice. To further identify the molecular mechanisms of MGF action on glucose metabolism, we conducted targeted metabolomics and transcriptomics studies of glycolyic and mitochondrial bioenergetics pathways in skeletal muscle. These data revealed that MGF increased glycolytic metabolites that were further augmented as glycolysis proceeded from the early to the late steps. Consistent with an MGF-stimulation of glycolytic flux there was a concomitant increase in the expression of enzymes catalyzing glycolysis. MGF also increased important metabolites in the tricarboxylic acid (TCA) cycle, such as $\alpha$-ketoglutarate and fumarate. Interestingly however, there was a reduction in succinate, a metabolite that also feeds into the electron transport chain to produce energy. MGF increased succinate clearance by enhancing the expression and activity of succinate dehydrogenase, leading to increased ATP production. At the transcriptional level, MGF induced mRNAs of mitochondrial genes and their transcriptional factors. Together, these data suggest that MGF upregulates mitochondrial oxidative capacity that likely drives the acceleration of glycolysis flux.
\end{abstract}

Keywords: mangiferin; glucose; glycolysis; TCA cycle; mitochondrial bioenergetics; metabolomics; transcriptomics

\section{Introduction}

The hallmark of diabetes is hyperglycemia. One of the main causes of hyperglycemia is inefficient or impaired glucose utilization in skeletal muscle, which can be exacerbated by chronic high caloric intake. Following uptake, glucose metabolism takes place in three pathways, glycogen synthesis, the pentose phosphate shunt and glycolysis. The last one generates pyruvate, a key metabolic branching point at which carbohydrate metabolism either goes through the anaerobic pathway to form lactate or the aerobic pathway to generate energy in mitochondria. While formation of lactate is a relatively fast way for glucose metabolism, the oxidative metabolism in mitochondria produces much more energy and is a more effective pathway to utilize carbohydrate nutrients and eliminate energy storage in form of, for instance, fat. 
The oxidative metabolism of carbohydrates in mitochondria is composed of two processes, the tricarboxylic acid (TCA) cycle and the electron transport chain (ETC). Pyruvate enters the TCA cycle mainly via two possible pathways, pyruvate decarboxylation by pyruvate dehydrogenase (PDH) to form acetyl-CoA, whose subsequent metabolites feed into the ETC for mitochondrial respiration to produce energy, or carboxylation by pyruvate carboxylase to form oxaloacetate, which participates in TCA cycle anaplerosis [1,2]. The majority of pyruvate enters the TCA cycle via the irreversible pyruvate decarboxylation by $\mathrm{PDH}$ [2]. In addition, there are two other irreversible reactions in the TCA cycle, citrate synthase (CS) catalyzed formation of citrate from acetyl-CoA and $\alpha$-ketoglutarate dehydrogenase ( $\alpha-\mathrm{KGDH}$ ) catalyzed formation of succinyl-CoA from $\alpha-\mathrm{KG}$. Based on the reversibility of catabolic reactions, the TCA cycle is thought to operate in two sequential segments, the first being from acetyl-CoA until $\alpha-K G$ and the second being from $\alpha-K G$ to oxaloacetate [3].

Impaired glycolysis and mitochondrial bioenergetics have been reported under pathological conditions such as obesity and diabetes [4]. Previous studies have identified several enzymatic steps that are dysregulated in diabetes in vivo or under hyperglycemic conditions in vitro. In particular, phosphofructose kinase (PFK) and glyceraldehyde 3-phosphate dehydrogenase (GAPDH) are down-regulated in terms of both expression and activity [5-12]. Significantly reduced resting metabolic flux through the TCA cycle and oxidative phosphorylation were reported in skeletal muscle of patients with type 2 diabetes and insulin resistant offspring of type 2 diabetic patients [13-15]. Reduced metabolites in the TCA cycle were also reported in high fat diet (HFD)-fed mice $[2,16]$. In parallel with reduced metabolic flux, mitochondrial DNA (mtDNA) and the transcription of mitochondrial genes are also suppressed in skeletal muscle in insulin-resistant individuals [17,18]. It has been proposed that correction of impaired catalytic steps in glycolysis and mitochondrial bioenergetics could mitigate hyperglycemia. Thus, there has been great interest in identifying mechanisms that increase glycolysis and mitochondrial bioenergetics, and based on such mechanisms identifying nutraceuticals/pharmaceuticals that can improve glucose homeostasis.

MGF is a C-glucosyl xanthone (C2- $\beta$-D-glucopyranosyl-1,3,6,7-tetrahydroxyxanthone) that is a predominant constituent in extracts of the mango plant Mangifera indica L. Recently, we reported that MGF can enhance insulin sensitivity and mitigate glucose intolerance in HFD-induced insulin resistant mice [19]. In addition, MGF enhanced glucose oxidation both in cultured C2C12 myotubes and, particularly interestingly, in skeletal muscle isolated from obese and insulin resistant mice induced by HFD feeding. We have also demonstrated that MGF activates PDH, which presumably directs glucose metabolism to the mitochondrial oxidative processes. However, detailed information on how MGF affects each step of glycolysis and mitochondrial oxidative metabolism remains unknown. In this study, we investigated the molecular mechanisms responsible for the metabolic beneficial effects of MGF by using targeted metabolomics and transcriptomics.

\section{Results}

\subsection{Profiling of Metabolites in Glucose Metabolism}

In our previous study, we found that MGF orally administered to mice on HFD was able to reduce plasma glucose and insulin levels [19]. We also found that MGF enhanced carbohydrate oxidation in skeletal muscle of mice fed either chow or HFD [19]. Thus, we proposed that the MGF's abilities of sensitizing insulin signaling and lowering glucose is, at least partially, due to MGF enhanced oxidative utilization of glucose in skeletal muscle. The processes of oxidative utilization of carbohydrates include glycolysis, and subsequent entry into the TCA cycle and the ETC. To understand how MGF affected these metabolic processes, we isolated quadriceps skeletal muscle from mice maintained on a normal low fat or HFD and were treated with or without MGF for 16 weeks, as previous reported [19] and analyzed metabolites in those skeletal muscle tissues.

Quantitative targeted metabolomics of several metabolic intermediates are listed in Table 1. Among all metabolites listed in Table 1, HFD significantly increased one metabolite, 
glucose-6-phosphate. Conversely, it significantly reduced several other metabolites in the glycolysis and the TCA cycle including phosphoenolpyruvate (PEP), pyruvate, $\alpha-K G$, succinate, fumarate and aspartate. Overall MGF significantly affected more metabolites in HFD-fed mice than in chow fed mice.

\subsection{MGF Accelerates Glycolysis}

\subsubsection{MGF Increases Glucose Metabolism via Glycolysis}

To more clearly demonstrate the effects of MGF in the context of metabolic pathways, we present significantly altered metabolites in bar graphs in the pathways (Figure 1). The first metabolite of glucose is glucose-6-phosphate, which can be metabolized by three pathways, glycogen synthesis, the pentose phosphate shunt, and glycolysis. Urindine diphosphate glucose (UDPG) is the product of glucose-6-phosphate and precursor of glycogen. MGF did not significantly affect UDPG in either chow or HFD-fed mice (Table 1), suggesting that MGF probably did not affect glycogen synthesis pathway (Figure 1). As to the pentose phosphate shunt, MGF did not significantly affect 6-phosphogluconate (an important intermediate metabolite), nor ribose-5-phosphate (an important terminal metabolite) (Table 1), suggesting that MGF probably did not exert any significant effects on this pathway (Figure 1). In contrast, MGF increased glucose 6-phosphate, F-1,6-bisphosphate, 3-phosphoglycerate and PEP in chow and/or HFD-fed mice. The magnitude of these differences became greater, i.e., the ratios (MGF/Ctl) increased, as glycolysis proceeded from the early steps to the late steps, indicating that MGF accelerated metabolic flux via the glycolysis pathway. The effects are more significant and profound in HFD-fed mice than in chow mice (Table 1, Figure 1).

\subsubsection{MGF Induces Some Enzymes in Glycolysis}

To further understand how MGF might alter the metabolites in glycolysis, we profiled mRNAs of those enzymes in muscle tissues from those chow or HFD-fed mice treated with or without MGF. HFD significantly reduced the mRNA of GAPDH, whereas HFD had no significant effect on the other glycolytic enzyme mRNAs examined (Figure 2). However, MGF treatment induced the mRNAs of three enzymes, HK, PFK, and GAPDH, in both chow and HFD-fed mice. Among these enzymes $G A P D H$ is the one on which MGF exerted the greatest effect (Figure 2). 
Table 1. Profiles of metabolites in quadriceps of mice.

\begin{tabular}{|c|c|c|c|c|c|c|c|c|c|c|}
\hline \multirow[b]{2}{*}{ Metabolite } & \multicolumn{4}{|c|}{ Chow } & \multicolumn{4}{|c|}{ HFD } & \multicolumn{2}{|c|}{ HFD/Chow (Ctl) } \\
\hline & Ctl & MGF & $\begin{array}{c}\text { Ratio } \\
\text { (MGF/Ctl) }\end{array}$ & $\begin{array}{c}* p(\text { MGF } \\
\text { Versus Ctl) }\end{array}$ & Ctl & MGF & $\begin{array}{c}\text { Ratio } \\
\text { (MGF/Ctl) }\end{array}$ & $\begin{array}{c}* p(\text { MGF } \\
\text { Versus Ctl) }\end{array}$ & Ratio & $* p$ \\
\hline UDPG & 7.95 & 7.63 & 0.96 & 0.115 & 6.40 & 5.89 & 0.92 & 0.316 & 0.80 & 0.098 \\
\hline Glucose-6-phosphate & 6.93 & 8.90 & 1.28 & 0.272 & 13.19 & 18.84 & 1.43 & 0.018 & 1.90 & 0.034 \\
\hline Fructose-1,6-bisphosphate & 44.17 & 58.90 & 1.33 & 0.099 & 54.28 & 76.40 & 1.41 & 0.043 & 1.23 & 0.451 \\
\hline 3-Phosphoglycerate & 26.03 & 34.09 & 1.31 & 0.089 & 28.39 & 46.18 & 1.63 & 0.030 & 1.09 & 0.445 \\
\hline Phosphoeolpyruate & 1.58 & 2.18 & 1.38 & 0.054 & 0.89 & 2.27 & 2.54 & 0.024 & 0.57 & 0.025 \\
\hline Pyruvate & 20.48 & 25.11 & 1.23 & 0.175 & 15.41 & 18.94 & 1.23 & 0.083 & 0.75 & 0.038 \\
\hline Lactate & 350.59 & 272.19 & 0.78 & 0.046 & 313.57 & 213.15 & 0.68 & 0.029 & 0.89 & 0.144 \\
\hline Glycerol-3-phosphate & 120.48 & 131.00 & 1.09 & 0.329 & 103.34 & 133.68 & 1.29 & 0.152 & 0.86 & 0.274 \\
\hline Ribose-5-phosphate & 0.39 & 0.43 & 1.09 & 0.216 & 0.44 & 0.47 & 1.05 & 0.688 & 1.13 & 0.406 \\
\hline 6-Phosphogluconate & 1.40 & 1.58 & 1.13 & 0.193 & 1.19 & 1.07 & 0.89 & 0.217 & 0.85 & 0.130 \\
\hline$\alpha$-Ketoglutarate & 0.45 & 0.67 & 1.48 & 0.043 & 0.28 & 0.62 & 2.20 & 0.041 & 0.63 & 0.035 \\
\hline Succinate & 50.81 & 31.23 & 0.61 & 0.038 & 36.96 & 21.85 & 0.59 & 0.032 & 0.73 & 0.050 \\
\hline Fumarate & 8.17 & 11.24 & 1.38 & 0.049 & 6.01 & 9.09 & 1.51 & 0.041 & 0.74 & 0.049 \\
\hline Malate & 43.89 & 48.31 & 1.10 & 0.121 & 35.64 & 40.45 & 1.13 & 0.322 & 0.81 & 0.084 \\
\hline Aspartate & 26.48 & 29.53 & 1.12 & 0.090 & 19.45 & 20.82 & 1.07 & 0.210 & 0.73 & 0.042 \\
\hline
\end{tabular}

C57BL6/J mice were fed chow or HFD and treated with MGF or without MGF for 16 weeks. Mice were then sacrificed after fasting for $12 \mathrm{~h}$ from $10 \mathrm{p} . \mathrm{m}$. to $10 \mathrm{a} . \mathrm{m}$. Their quadriceps were collected and snap frozen in liquid $\mathrm{N}_{2}$. Metabolites were analyzed by the metabolomics study. Values are average, $n=6 .{ }^{*} p<0.05$ is considered significant. HFD, high fat diet; MGF, mangiferin; Ctl, control; UDPG, urindine diphosphate glucose. 


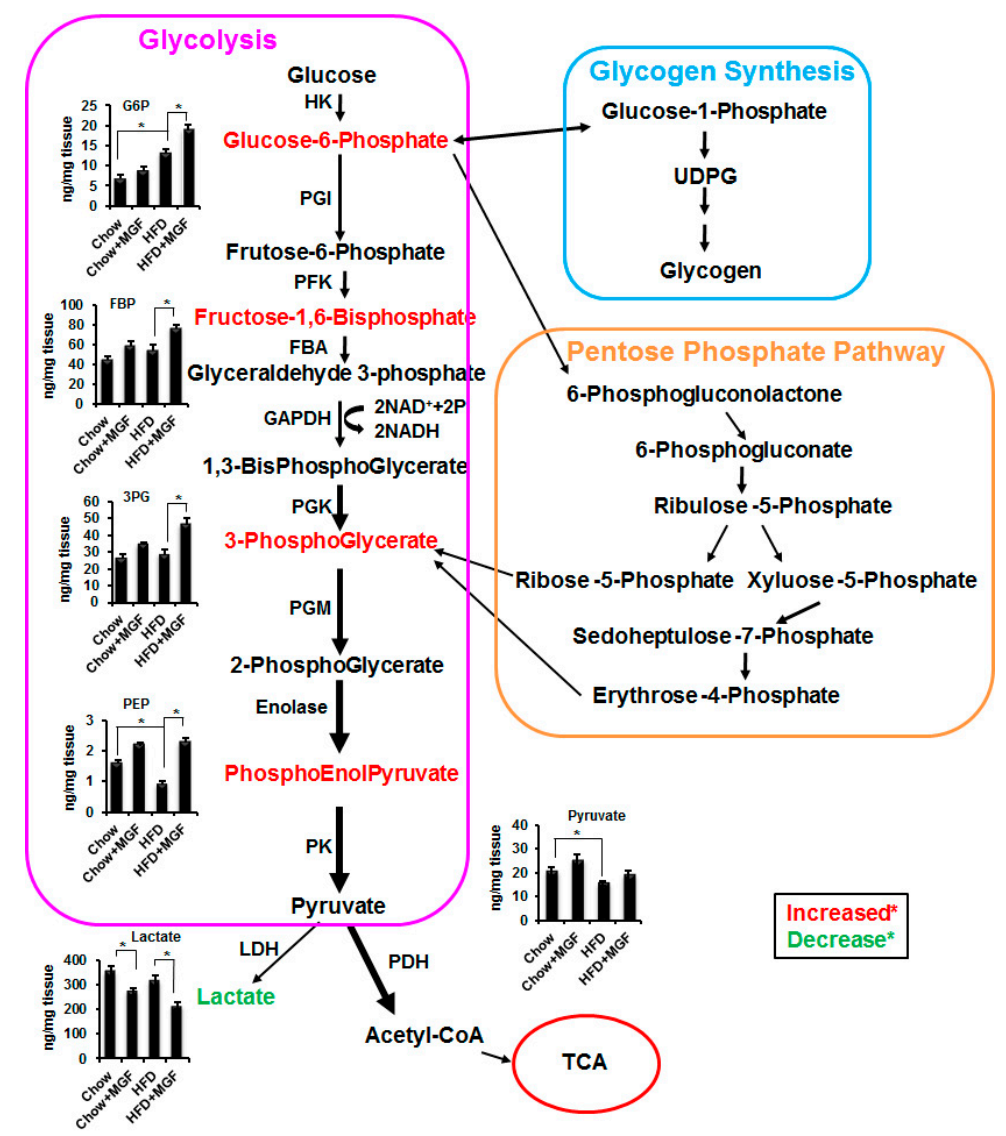

Figure 1. MGF accelerates glycolysis. Glucose metabolic pathways include glycogen synthesis, pentose phosphate shunt and glycolysis. Arrows in the glycolysis pathway indicate mangiferin directed metabolic flux from one product to the next. Arrows in other pathways mean change of one metabolic product to the other. Significantly altered metabolites are presented in bar graphs. Values are average \pm SEM $(n=6) .{ }^{*} p<0.05$. HK, Hexose kinase; PGI, Phosphoglucose isomerase; PFK, Phosphofructokinase; FBA, Fructose-1,6-bisphosphate aldolase; GAPDH, Glyceraldehyde 3-phosphate dehydrogenase; PGK, Phosphoglycerate kinase; PGM, Phosphoglycerate mutase; PK, Pyruvate kinase; PEP, phosphoenolpyruvae; LDH, lactate dehydrogenase; PDH, pyruvate dehydrogenase; UDPG, urindine diphosphate glucose.

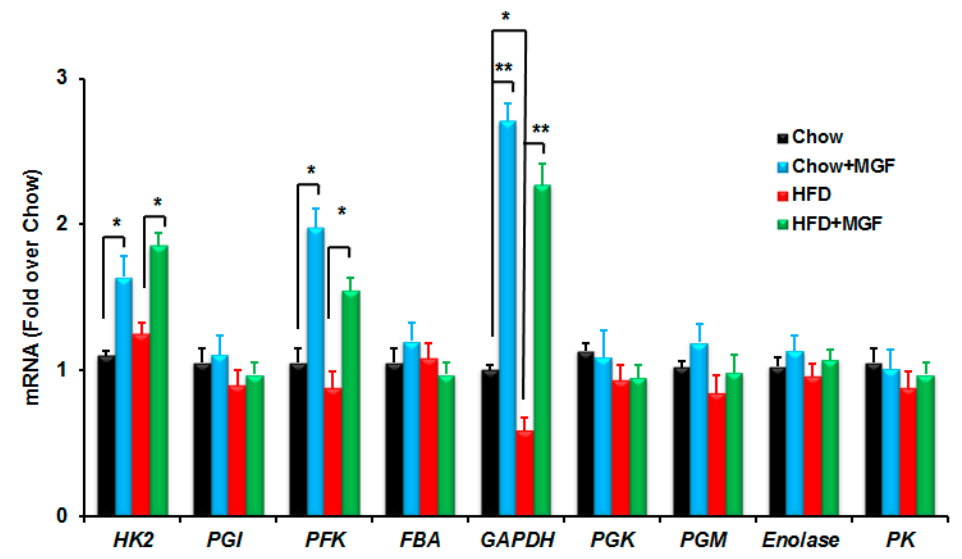

Figure 2. MGF induces mRNAs of some enzymes participating in glycolysis. Profiles of mRNAs of glycolytic enzymes in quadriceps from mice treated with chow or HFD \pm MGF for 16 weeks. Values are average $\pm \operatorname{SEM}\left(n=6\right.$ /group). ${ }^{*} p<0.05,{ }^{* *} p<0.01$. 


\subsection{MGF Enhances Mitochondrial Bioenergetics}

\subsubsection{MGF Enhances Metabolism in the TCA Cycle}

Accelerated glycolysis by itself would lead to an increase in the end product, pyruvate. However, there was no significant increase in pyruvate levels in the MGF treated mice, suggesting a more rapid clearance of pyruvate. Pyruvate can be catabolized to several products via different pathways. Pyruvate can be converted to lactate by lactate dehydrogenase (LDH) via the anaerobic pathway or enter the TCA cycle to be metabolized via the aerobic pathway. Figure 1 shows that there is significantly less lactate produced in MGF treated mice, suggesting that the MGF-increased clearance of pyruvate was not via the anaerobic pathway in the cytosol, but rather via the TCA cycle occurring in mitochondria.

In mitochondria, the majority of pyruvate is catabolized to acetyl CoA by PDH [2]. Acetyl CoA is then further metabolized to $\alpha-K G$, which is a marker of the TCA flux during the first segment. We have previously reported that MGF activates PDH [19], and therefore we hypothesized that MGF accelerated the first segment of the TCA cycle. Indeed, MGF significantly increased $\alpha-\mathrm{KG}$, by about $150 \%$ in chow mice and by $220 \%$ in HFD muscle (Table 1, Figure 3). Interestingly, MGF elevated $\alpha-K G$ did not result in any increase in the subsequent metabolite of $\alpha-K G$, succinate. Rather, MGF significantly reduced succinate by about $40 \%$ in chow and HFD muscles. On the other hand, MGF significantly increased fumarate formed from succinate by SDH by $138 \%$ in chow mice and by $151 \%$ in HFD muscle (Table 1, Figure 3). Fumarate is in rapid exchange with other four-carbon intermediates, malate and oxaloacetate, which can be reversibly converted to aspartate. MGF increased all these metabolites in the second segment of the TCA cycle, albeit some increases were insignificant.

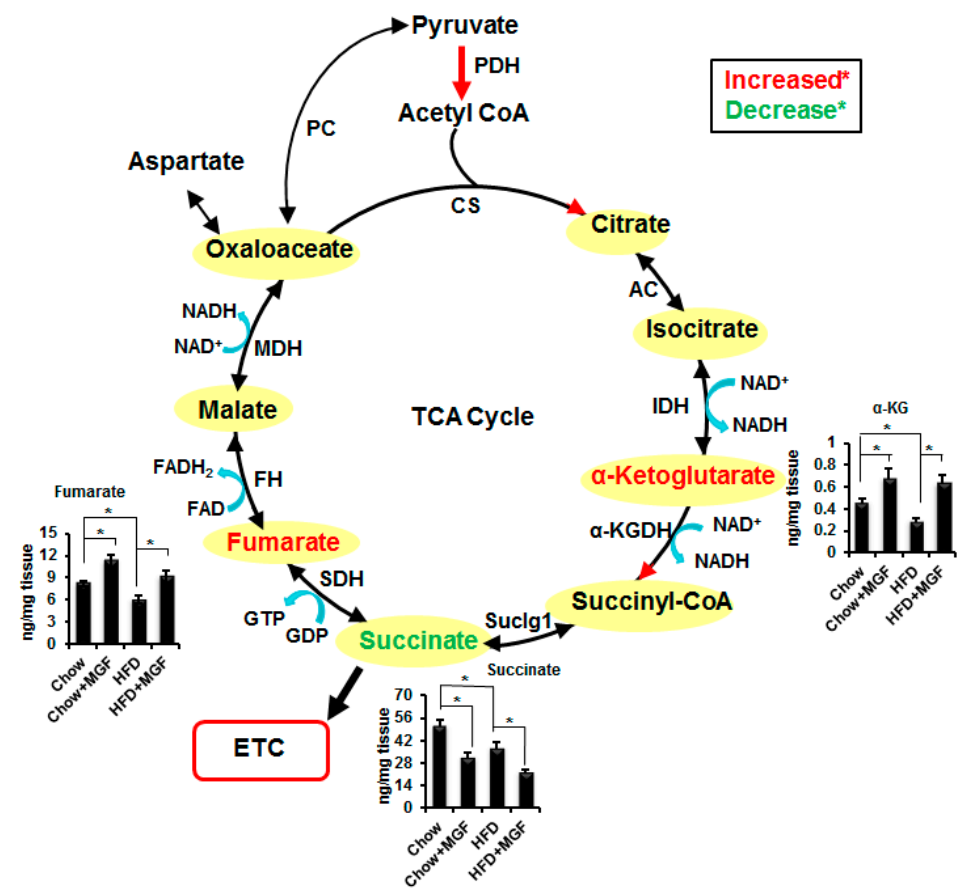

Figure 3. MGF modulates metabolite profiles in the TCA cycle. One-end arrows mean unidirectional and two-end arrows mean bidirectional. Significantly altered metabolites are presented in bar graphs. Values are average $\pm \operatorname{SEM}(n=6) .{ }^{*} p<0.05$. PC, pyruvate carboxylase; PDH, pyruvate dehydrogenase; $\mathrm{CS}$, citrate synthase; AC, Aconitase; IDH, isocitrate dehydrogenase; $\alpha-\mathrm{KGDH}, \alpha$-ketoglutarate dehydrogenase; Suclg, succinyl coenzyme A synthetase; $\mathrm{SDH}$, succinate dehydrogenase; $\mathrm{FH}$, fumarase; $\mathrm{MDH}$, malate dehydrogenase; $\alpha-\mathrm{KG}, \alpha$-ketoglutarate. 


\subsubsection{MGF Enhances SDH Activity and Overall Energy Production in Mitochondria}

Succinate is an intermediate between $\alpha-K G$ and fumarate that were both increased, suggesting that the decrease in succinate levels was due to enhanced succinate consumption. MGF significantly increased the activity of SDH in mitochondrial isolated from quadriceps by $63 \%$ and $86 \%$ in mice fed with chow and HFD, respectively (Figure 4a). To confirm these in vivo effects, we treated C2C12 myotubes with various concentrations of MGF for about $4 \mathrm{~h}$ and isolated mitochondria from those cells and measured SDH activity. MGF also significantly increased SDH activity in those cells in a dose-dependent manner (Figure $4 b$ ).

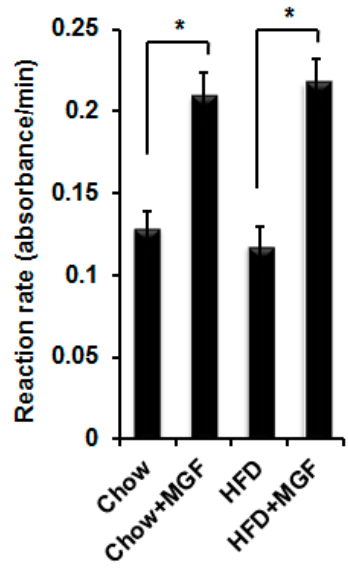

(a)

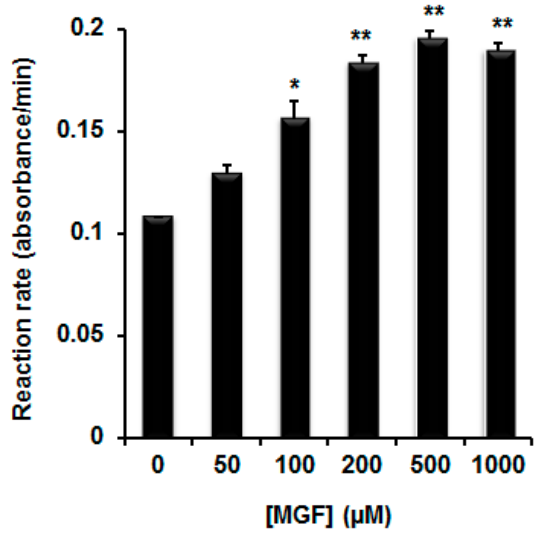

(b)



(c)

Figure 4. MGF enhances SDH activity and increases ATP production. Mitochondria were isolated from quadriceps of mice treated with chow or HFD \pm MGF for 16 weeks ( $n=6 /$ group) (a) or C2C12 myotubes treated with various concentrations of MGF for $4 \mathrm{~h}(\mathbf{b})$. In vitro experiment presented in (b) was repeated for three times. The comparisons are between the rate at each MGF concentration with the rate when $[\mathrm{MGF}]=0$; (c) ATP production in freshly isolated mitochondria from quadriceps of mice treated with chow or HFD \pm MGF for 16 weeks $\left(n=6\right.$ /group). Values are average \pm SEM. ${ }^{*} p<0.05$, ** $p<0.01$.

Among the metabolites in the TCA cycle, succinate is the only one that enters the ETC to participate in a chain of oxidative reactions to ultimately produce energy, ATP. SDH is also the mitochondrial complex II in the ETC. SDH activity is a marker of mitochondrial oxidative capacity and positively related to energy production [20]. Thus, we measured ATP production in freshly isolated mitochondria from snap frozen quadriceps. HFD significantly reduced ATP production (Figure 4c). MGF significantly increased ATP production by about $150 \%$ in chow mice. Moreover, MGF reversed the reduction in ATP in HFD mice and increased the level of ATP slightly higher than that in chow control mice.

\subsubsection{MGF Induces Mitochondrial Biogenesis}

To investigate whether MGF has any effects on the expressions of mitochondrial genes, we profiled mRNAs of all enzymes in the TCA cycle and the ETC from the quadriceps skeletal muscle. HFD feeding caused reduction in most of mitochondrial genes, although most of these changes did not reach statistical significance (Figure $5 a, b$ ). On the other hand, MGF significantly induced several genes in the TCA cycle and the ETC in chow and/or HFD-fed mice (Figure 5a,b). These genes included IDH, $\alpha K G D H, S D H, C o x I, C y t-c, C O X I V$ and ATP5g1. The greatest effects occur in SDH, which could be a factor contributing MGF enhanced catabolism of succinate to fumarate. 

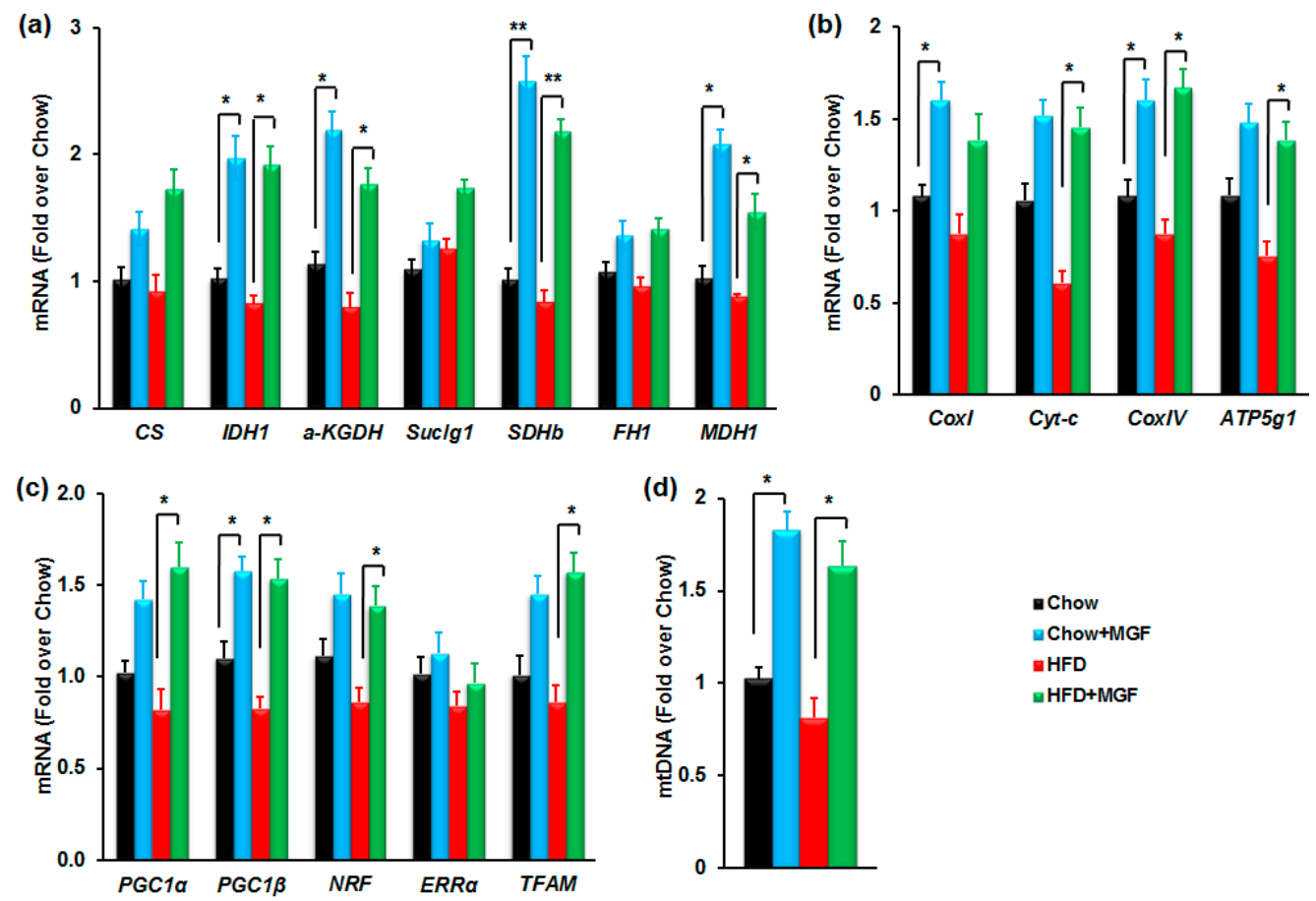

Figure 5. MGF induces mitochondrial biogenesis. (a-c) Profiles of mRNAs of mitochondrial genes $(\mathbf{a}, \mathbf{b})$ and transcriptional factors (c) in quadriceps of mice treated with chow or HFD \pm MGF for 16 weeks ( $n=6$ /group); (d) Relative mitochondria DNA contents in quadriceps of mice treated with chow or $\mathrm{HFD} \pm \mathrm{MGF}$ for 16 weeks. Values are average $\pm \operatorname{SEM}(n=6) .{ }^{*} p<0.05,{ }^{* *} p<0.01$.

Mitochondrial genes are tightly regulated by several transcription factors including transcriptional factor A (TFAM), nuclear respiratory factor 1 (NRF1) and estrogen-related receptor $\alpha(E R R \alpha)$, and the co-factors of these transcriptional factors, peroxisome proliferator-activated receptor- $\gamma$ coactivator (PGC)-1 $\alpha$ and PGC-1 $\beta$ [21-26]. MGF significantly increased all of those transcriptional factors, except $E R R \alpha$, in chow or HFD-fed mice (Figure 5c), indicating that MGF upregulates those enzymes in the TCA cycle and the ETC at the transcriptional level. PGC-1 $\alpha$ is positively correlated to mtDNA. Therefore, we measured mtDNA contents in mitochondria isolated from those muscle tissues. MGF almost doubled mtDNA in both chow and HFD-fed mice (Figure 5d). Together, these results indicate that MGF induces mitochondrial biogenesis.

\section{Discussion}

Obese, insulin resistant and diabetic patients display impaired glycolysis and dysfunctional mitochondrial oxidation $[4,13-15,17,18]$, which are major contributors to impaired glucose disposal/utilization and hence to hyperglycemia. Pharmaceutical and/or nutraceutical agents that can improve glycolysis and mitochondrial oxidative capacity have the potential for therapeutic value in treating diet induced obesity and insulin resistance. Using a HFD mouse model of obesity and insulin resistance, we previously identified a natural compound, MGF, that was able to correct HFD induced glucose intolerance and insulin resistance by enhancing glucose utilization [19]. Using metabolite and transcriptomic profiling we have found that MGF gradually increased metabolic flux in glycolysis, which was facilitated by MGF induced enzymes in glycolysis. In mitochondria, MGF directs pyruvate metabolism in sequence from the first segment to the second. In such manner MGF enhances oxidative metabolism, resulting in increased production of energy, ATP. MGF does so by upregulating transcription of mitochondrial genes and enhancing the activity of SDH.

Glucose-6-phosphate is the first metabolite in glucose metabolism. HFD feeding almost doubled glucose-6-phosphate probably due to HFD blunted metabolism at the subsequent metabolic steps, as 
there was gradually augmented reduction in metabolites from the early steps to the later. Near the end of glycolysis, HFD caused significant reduction in PEP and pyruvate (Table 1, Figure 1). On the contrary, MGF gradually and significantly increased metabolites and the increases were augmented from the early steps to the later (Table 1, Figure 1). These results indicate that MGF is able to counteract the inhibitory effects of HFD on glycolysis.

The conversion of glucose to glucose-6-phosphate is catalyzed by HK. Previously Sellamuthu et al. [27] showed that MGF increased HK activity in liver of streptozotocin induced diabetic rats. Here we show that MGF induces HK expression in muscle of both chow and HFD-fed mice (Figure 2). In glycolysis, glucose-6-phosphate is subsequently converted to fructose-1,6-bisphosphate by PFK. While the effects of HFD on the activity of PFK is uncertain [28-30], diabetes reduces the expression and activity of PFK [31]. Deficiency in PFK is associated with impaired insulin secretion and insulin resistance in human [5,6,8,32]. MGF induces PFK (Figure 2) and therefore has the possible capability of mitigating PFK deficiency in diabetes. MGF also significantly induced the mRNA of GAPDH, which is a key enzyme in the glycolytic conversion of glucose to pyruvate and has been considered to play a regulatory role in setting the glycolytic rate [33,34]. The reports on the effects of HFD feeding on expression of GAPDH are inconsistent probably due to tissue specificity [35-37]. Here we show that HFD significantly reduced GAPDH mRNA in skeletal muscle (Figure 2). More importantly, MGF treatment caused more than 2-fold induction in mRNA of GAPDH in both chow and HFD-fed mice (Figure 2).

The end product of glycolysis is pyruvate, which can be converted to lactate via the anaerobic process or enter mitochondria to be metabolized by the aerobic process. While HFD did not cause significant change in lactate, it significantly reduced several key metabolites in the TCA cycle (Table 1 , Figure 3), which was also reported by others $[2,16]$. MGF modulated those metabolites in a biologically important manner. Pyruvate is converted from PEP by PK. MGF increased PEP, but did not suppress PK. One would expect that there could be similar magnitude of increase in pyruvate in MGF treated mice. However, MGF only caused mild and insignificant increase in pyruvate, suggesting that MGF probably enhanced pyruvate consumption, either by increasing lactate production or by enhancing oxidative metabolic flux in the TCA cycle. MGF significantly reduced lactate in muscle tissues of both chow and HFD-fed mice (Table 1, Figure 1), which recapitulates the effects of MGF on lactate in cultured C2C12 myotubes shown in our previous study [19]. We further demonstrated that MGF reduced lactate production not by inhibiting pyruvate carboxylase (lactate dehydrogenase), but by enhancing PDH activity, shuttling pyruvate to mitochondria. In mitochondria PDH catalyzes the irreversible conversion of pyruvate to acetyl-CoA, which will be irreversibly converted to citrate by citrate synthase (CS). These unidirectional reactions make commitment to the flow of the TCA cycle to produce the end product of the first segment of the TCA cycle, $\alpha-K G$. The fact that MGF increased $\alpha-K G$, as shown in this study (Table 1, Figure 3), confirms that MGF enhances PDH and accelerates the metabolism in the first segment of the TCA cycle. This enhancement is also supported by MGF increased CS and, more significantly, IDH (Figure 5a).

Subsequently, $\alpha-K G$ is irreversibly converted to succinyl-CoA by $\alpha-K G D H$. MGF significantly increased $\alpha-\mathrm{KGDH}$, presumably increasing succinyl-CoA, the starting point of the second segment of the TCA cycle, leading to the production of oxaloacetate. All of the reactions in the second segment of the TCA cycle are reversible and some of the metabolites are in rapid exchange with each other. MGF increased most metabolites in the second segment of the TCA cycle, except succinate. Succinate is a unique metabolite. It is the only metabolite in the TCA cycle that also feeds in the ETC and participates in oxidative phosphorylation. Accumulation of succinate is a marker of anaerobic metabolism [3]. MGF significantly reduced succinate, which supports the idea that MGF enhances oxidative metabolism.

MGF eliminates succinate probably by enhancing SDH, as MGF upregulates SDH at both the expression and the activity levels (Figures $4 a, b$ and $5 a$ ). SDH is the only enzyme that is a component of both the TCA cycle and the ETC. In the ETC, it is named complex II. SDH catalyzes the oxidation of succinate to fumarate and uses the electrons derived from this oxidation to catalyze the reduction 
of ubiquinione to ubiquinol. These electrons are passed to complex III and then complex IV, thereby contributing to the establishment of the electrochemical gradient across the mitochondria inner membrane for ATP synthesis [38]. Among all mitochondrial complexes, complex II loses activity most rapidly after death and on inappropriate storage, and therefore its activity is considered as a marker of the integrity of the tissue and the reliability of respiratory chain enzymology [20]. Mutation of SDH gene results in mitochondrial respiratory chain deficiency [39]. Reduced SDH expression and activity were reported in obese and diabetic patients [18]. Here we show that MGF upregulates SHD at both expression and activity levels in quadriceps of not only wild type mice but also, importantly, in obese and insulin resistant mice (Figures $4 a, b$ and $5 a$ ). MGF was also shown to enhance SDH activity in soleus and tibialis of obese rats, and to increase SDH activity in heart of experimentally induced myocardial infarcted rats $[40,41]$.

The effects of MGF on mitochondria are not limited to SDH. In addition to SDH, MGF was shown to increase the activities of other important enzymes in the TCA cycle and the ETC, including $\alpha-\mathrm{KGDH}$, $\mathrm{MDH}$ and cytochrome c [41]. At the expression level, here we found that MGF induced mRNAs of most enzymes in the TCA cycle and the ETC (Figure 5a,b). Furthermore, MGF induced several important mitochondrial transcriptional factors and their co-factors including TFAM, NRF1, PGC-1 $\alpha$ and PGC-1 $\beta$ (Figure $5 \mathrm{c}$ ), as well as increasing mitochondrial DNA content (Figure $5 \mathrm{~d}$ ). Taking together, these results indicate that MGF enhanced overall mitochondrial oxidative capacity, which probably drives the accelerated metabolic flux in glycolysis (Figure 1).

In addition to accelerating glycolysis, MGF has been shown to reduce glucose 6-phosphate and fructose bisphosphatase and therefore inhibit gluconeogenesis [27]. These effects, together, suggest that MGF could be an effective anti-hyperglycemia and anti-diabetes agent. Furthermore, we and others have shown that MGF has anti-hyperlipidemia effects in rodents [19,42-47], and impressively in humans [48]. As a natural compound, MGF has been shown to uniquely possess multi-beneficial effects in mitigating metabolic disorders. The drawback is that it has very low bioavailability (1.2\%) [49-51] and therefore the dose required for MGF to exert significant effects is high. Nevertheless, the structural features of MGF allow chemical modifications and MGF has the potential to be developed into an effective nutraceutical/pharmaceutical for the treatment of complex metabolic syndrome.

\section{Materials and Methods}

Chemical reagents were purchased from Sigma-Aldrich (St. Louis, MO, USA) unless specified.

\subsection{Cell Culture}

C2C12 myoblast cells were maintained in DMEM High Glucose (Hyclone, Logan, UT, USA), supplemented with $1 \%$ penicillin and streptomycin (GIBCO, Carlsbad, CA, USA) and 10\% FBS. C2C12 cells were differentiated into myotubes in the above DMEM medium containing $2 \%$ horse serum (replacing 10\% FBS) for 4-6 days after cells reached confluence.

\subsection{Animal Experiments}

Wild type C57BL6/J mice at age of 5-6 weeks were purchased from Jackson laboratory (Bar Harbor, ME, USA). All studies were approved by and performed in compliance with the guidelines of the Einstein Institutional Animal Care and Use Committee. Chow diet (CD) (providing 28.5\% (Kcal) protein, $13.5 \%$ fat and $58 \%$ carbohydrates) was obtained from Purina LabDiet, Framingham, MA, USA. HFD (providing 20\% (Kcal) protein, $60 \%$ fat and 20\% carbohydrates) was obtained from Research Diets, New Brunswick, NJ, USA. MGF was obtained from CTMedChem (Bronx, NY, USA) and administered orally via food. $C D$ and HFD were ground into fine powder and mixed with pure MGF. The mixtures were re-pelleted and administered to individually housed mice. Mice were fed ad libitum with CD or $\mathrm{HFD} \pm$ MGF (MGF dose, $400 \mathrm{mg} / \mathrm{kg}$ body weight) and water for 16 weeks. Mice were then sacrificed after 12 -h fasting from 10 p.m. to 10 a.m. Quadriceps were collected, snap frozen in liquid $\mathrm{N}_{2}$ and stored at $-80^{\circ} \mathrm{C}$. 


\subsection{Metabolite Profiling}

Metabolite profiling was conducted at the Einstein Stable Isotope \& Metabolomics Core. There are two main processes. One is sample preparation and the other is LC/MS/MS analysis. Tissue samples were homogenized with a mixture of methanol, acetonitrile and water (methanol:acetonitrile:water $=2: 2: 1$ (v:v:v)) with tissue factor of 6 (to tissue weight). Internal standard (1.8 $\mu \mathrm{g}$ of UC13-succinate) was spiked to each sample before extraction. The samples were homogenized and centrifuged. The supernatant was transferred to a glass tube for lyophilization. The residue was reconstituted in $265 \mu \mathrm{L}$ of $5 \mathrm{mM}$ ammonium fumarate in acetonitrile solution (acetonitrile:water $=6: 4(v: v)$ ).

LC/MS/MS (Waters Xevo TQ) analysis was performed to quantitate metabolites using similar method described previously [52]. Chromatographic analysis, prior to targeted and multiple reaction monitoring (MRM) mass spectrometric analyses, was performed using an Aquity UPLC using a Waters BEH amide $1.7 \mu \mathrm{m}$ column $2.1 \times 100 \mathrm{~mm}$, and an acidic mobile phase containing an acetonitrile/water gradient, as per [52]. Samples were bracketed in between calibration standards and linear regression was performed for quantitation.

\section{4. $m R N A$ Measurement by Real Time qRT-PCR}

Total RNA from muscle tissues was extracted with Trizol. One $\mu \mathrm{g}$ of total RNA was used to synthesize cDNA with Reverse Transcriptase and OligodT from Invitrogen (18080051, Carlsbad, CA, USA). Real time PCR using the Sybrgreen master mix (Applied Biosystems, Foster City, CA, USA) was performed by a 7900HT PCR machine from Applied Biosystems. The level of mRNA was calculated versus $\beta$-actin transcript and expressed as fold over control. All primers used for real time qRT-PCR are listed in Table 2.

Table 2. Mouse primers for real time qRTPCR.

\begin{tabular}{|c|c|c|}
\hline Gene & Forward Sequence from $5^{\prime}$ to $3^{\prime}$ & Reverse Sequencefrom $5^{\prime}$ to $3^{\prime}$ \\
\hline HK2 & TGATCGCCTGCTTATTCACGG & AACCGCCTAGAAATCTCCAGA \\
\hline PGI & TGGCCCAGACTGAGGCCCTG & CTCTAGGTGTCTTTATTCTA \\
\hline PFK & CAGATCAGTGCCAACATAACCAA & CGGGATGCAGAGCTCATCA \\
\hline$F B A$ & AGCCTTCTGAGAAGGATGCTC & GTCCAGCATGAAGCAGTTGAC \\
\hline GAPDH & ACTGGCATGGCCTTCCG & CAGGCGGCACGTCAGATC \\
\hline PGK1 & GGTCTTGCCAAAATGTCGCT & TCCAATCGAATCCTCGTGTC \\
\hline$P G M b$ & TGCCCGTCGCCATGGCTGCC- & CGGTGGGACATCATAAGATC- \\
\hline Enolase1 & GCCGGCTTTACGTTCACCTC & GTTGAAGCACCACTGGGCAC \\
\hline CS & AAGGACGAGGCAGGATGAG & TGCAGCTGTAGCTCTCTCCC \\
\hline Idh1 & ATTGGTGGCATCACGATTCT & TGGAGATGCAAGGAGATGAA \\
\hline$A K G D H$ & AGTGGTGGTGGGTAAGTGGA & GCAAAACTTGATCCTCTCGG \\
\hline Suclg1 & TGTTTCCGAGAGGCTGTGTA & CAACCATGGTCTCCAGCAG \\
\hline$S D H b$ & GTCTGTGCCCCTCGACAG & TGACGTCAGGAGCCAAAAT \\
\hline FH1 & ACACGGAAGGAATTTTGGCT & ACCATGTACCGCGCACTC \\
\hline MDH1 & TGCTCCAGTCACAAGGACTC & GACTGCTGGAGACTGCCTTT \\
\hline $\operatorname{CoxI}(\mathrm{Ndufa9})$ & TAAGGGATGAAGGTCCGATG & GATCCAGATGCCGTAGGAAA \\
\hline Cyc (cytochrome c) & GGAGGCAAGCATAAGACTGG & TCCATCAGGGTATCCTCTCC \\
\hline CoxIV & AGAAGGAAATGGCTGCAGAA & GCTCGGCTTCCAGTATTGAG \\
\hline Atp5g1 & GCTGCTTGAGAGATGGGTTC & AGTTGGTGTGGCTGGATCA \\
\hline$P G C-1 \alpha$ & AAGTGTGGAACTCTCTGGAACTG & GGGTTATCTTGGTTGGCTTTATG \\
\hline$P G C-1 \beta$ & TGCTGCTGTCCTCAAATACG & TGGAGACTGCTCTGGAAGGT \\
\hline NRF & CGGAGTGACCCAAACTGAAC & GATGACCACCTCGACCGTTT \\
\hline$E R R \alpha$ & ACTGCCACTGCAGGATGAG & CACAGCCTCAGCATCTTCAA \\
\hline TFAM & CCAAAAAGACCTCGTTCAGC & ATGTCTCCGGATCGTTTCAC \\
\hline B-actin & CATGGAGTCCTGTGGCATC & AGCACTGTGTTGGCGTAC \\
\hline
\end{tabular}




\subsection{Mitochondria Isolation}

Mitochondria were isolated from muscle tissues using previously described method [53,54] with modified buffer (250 mM sucrose, 1 mM EGTA, 10 mM HEPES, and 5 g/L BSA, pH 7.5). Mitochondrial pellets were suspended, and mitochondria were permeabilized by a freeze-thaw procedure before used to evaluate the activity of mitochondrial membrane complexes like complex II (SDH) $[53,54]$.

\subsection{Measurement of Mitochondrial Complex II Activity}

Complex II specific activity was measured by following the decline in absorbance at $600 \mathrm{~nm}$ due to the reduction of dichlorophenol indophenols. Two hundred and twenty five microliters of the reaction

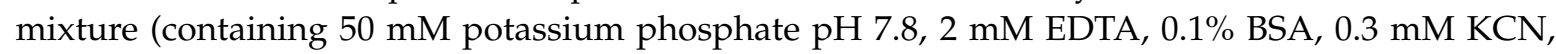
$50 \mathrm{mM}$ dichlorophenol indophenol, $3 \mu \mathrm{M}$ rotenone, $1 \mu \mathrm{M}$ antimycin A, $2 \mathrm{mmol} / \mathrm{L}$ ATP, $50 \mu \mathrm{M}$ CoQ1 and $45 \mu \mathrm{g}$ mitochondria protein) was loaded onto 96 -well plate and equilibrated at $30{ }^{\circ} \mathrm{C}$ for $5 \mathrm{~min}$. The reaction was started by addition of $25 \mu \mathrm{L}$ sodium succinate at the final concentration of $10 \mathrm{mM}$ and the enzyme-catalyzed reduction of dichlorophenol indophenol was monitored as absorbance at $600 \mathrm{~nm}$ for $5 \mathrm{~min}$. Graphs of absorbance versus time were plotted and reaction rates were calculated.

\subsection{Measurement of ATP Production}

ATP production was measured by using the method previously described [55], with some modifications. We reduced the total volume of the reaction mixture from $1 \mathrm{~mL}$ to $250 \mu \mathrm{L}$ and the reaction was carried out in 96-well plates.

\subsection{Mitochondrial DNA (mtDNA)}

Mitochondrial DNA content was determined according to the protocol described by Ajaz et al. [56]. It was quantified by real-time quantitative PCR.

\subsection{Statistical Analysis}

Group measurements were expressed as average \pm SEM. One-way ANOVA was used to test for differences among groups. When either a significant $(p<0.05)$ or a marginal $(0.05<p<0.1)$ effect was observed, two-tailed Student's $t$ tests were conducted to analyze the significance of the differences between two specific groups. $p<0.05$ was considered significant.

\section{Conclusions}

In this study, we report that MGF significantly increased metabolites in glycolysis, and the increases augmented from the early steps to the late steps, indicating that MGF accelerated glycolytic flux, and this was supported by MGF induced mRNAs of several enzymes catalyzing glycolysis. MGF enhanced overall metabolism in the TCA cycle in a direction from citrate to oxaloacetate. It increased most of metabolites in the TCA cycle, but reduced succinate. MGF enhanced conversion of succinate to fumarate, which occurs not only in the TCA cycle but also in the ETC, by upregulating SDH at both the activity and the expression levels. At the expression level, MGF upregulated not only mtDNA contents, but also mRNAs of most of mitochondrial genes and their transcriptional factors. Overall, MGF upregulated mitochondrial oxidative capacity, which was indicated by MGF-increased ATP production. This study provides molecular understanding of how MGF stimulates carbohydrate oxidation and thereby mitigates hyperglycemia and insulin resistance. As a natural compound, MGF has great potential to be developed into a nutraceutical/pharmaceutical to combat diabetes and obesity.

Acknowledgments: This work was supported by the grants that Yuling Chi received from the American Heart Association (0735066N), from American Diabetes Association (1-11-JF-06), and partially from NIH/NIDDK (DK41296 and DK020541).

Author Contributions: Yuling Chi conceived and designed the experiments; Zhongbo Liu, Pasha Apontes and Ekaterina V. Fomenko performed the experiments; Yuling Chi, Zhongbo Liu, Ekaterina V. Fomenko, Nan Chi 
and Irwin J. Kurland analyzed the data; Yuling Chi wrote the paper; Victor L. Schuster and Jeffrey E. Pessin edited paper.

Conflicts of Interest: The authors declare no conflict of interest.

\section{Abbreviations}

$\begin{array}{ll}\text { TCA } & \text { Tricarboxylic acid } \\ \text { ETC } & \text { Electron transport chain } \\ \text { PDH } & \text { Pyruvate dehydrogenase } \\ \text { CS } & \text { Citrate synthase } \\ \alpha-\text { KG } & \alpha \text {-Ketoglutarate } \\ \alpha-\text { KGDH } & \alpha \text {-Ketoglutarate dehydrogenase } \\ \text { PFK } & \text { Phosphofructose kinase } \\ \text { GAPDH } & \text { Glyceraldehyde 3-phosphate dehydrogenase } \\ \text { HFD } & \text { High fat diet } \\ \text { MGF } & \text { Mangiferin } \\ \text { UDPG } & \text { Urindine diphosphate glucose } \\ \text { PEP } & \text { Phosphoenolpyruvate } \\ \text { HK } & \text { Hexose kinase } \\ \text { PGI } & \text { Phosphoglucose isomerase } \\ \text { PFK } & \text { Phosphofructokinase } \\ \text { FBA } & \text { Fructose-1,6-bisphosphate aldolase } \\ \text { PGK } & \text { Phosphoglycerate Kinase } \\ \text { PGM } & \text { Phosphoglycerate mutase } \\ \text { PK } & \text { Pyruvate kinase } \\ \text { LDH } & \text { Lactate dehydrogenase } \\ \text { PC } & \text { Pyruvate carboxylase } \\ \text { CS } & \text { Citrate synthase } \\ \text { AC } & \text { Aconitase } \\ \text { IDH } & \text { Isocitrate dehydrogenase } \\ \text { Sucl } & \text { Succinyl coenzyme A synthetase } \\ \text { SDH } & \text { Succinate dehydrogenase } \\ \text { FH } & \text { Fumarase } \\ \text { MDH } & \text { Malate dehydrogenase } \\ \text { Atp5g1 } & \text { ATP synthase subunit 5g1 } \\ \text { TFAM } & \text { Transcriptional factor A } \\ \text { NRF1 } & \text { Nuclear respiratory factor 1 } \\ \text { ERR } \alpha & \text { Estrogen-related receptor } \alpha \\ \text { PGC-1 } \alpha & \text { Peroxisome proliferator-activated receptor- } \gamma \text { coactivator-1 } \alpha \\ \text { PGC-1 } \beta & \text { Peroxisome proliferator-activated receptor- } \gamma \text { coactivator-1 } \\ \text { mtDNA } & \text { Mitochondrial DNA } \\ \text { PBS } & \end{array}$

\section{References}

1. Owen, O.E.; Kalhan, S.C.; Hanson, R.W. The key role of anaplerosis and cataplerosis for citric acid cycle function. J. Biol. Chem. 2002, 277, 30409-30412. [CrossRef] [PubMed]

2. Kowalski, G.M.; de Souza, D.P.; Burch, M.L.; Hamley, S.; Kloehn, J.; Selathurai, A.; Tull, D.; O'Callaghan, S.; McConville, M.J.; Bruce, C.R. Application of dynamic metabolomics to examine in vivo skeletal muscle glucose metabolism in the chronically high-fat fed mouse. Biochem. Biophys. Res. Commun. 2015, 462, 27-32. [CrossRef] [PubMed]

3. Chinopoulos, C. Which way does the citric acid cycle turn during hypoxia? The critical role of $\alpha$-ketoglutarate dehydrogenase complex. J. Neurosci. Res. 2013, 91, 1030-1043. [CrossRef] [PubMed]

4. Abdul-Ghani, M.A.; DeFronzo, R.A. Mitochondrial dysfunction, insulin resistance, and type 2 diabetes mellitus. Curr. Diabetes Rep. 2008, 8, 173-178. [CrossRef] 
5. Ristow, M.; Carlqvist, H.; Hebinck, J.; Vorgerd, M.; Krone, W.; Pfeiffer, A.; Muller-Wieland, D.; Ostenson, C.G. Deficiency of phosphofructo-1-kinase/muscle subtype in humans is associated with impairment of insulin secretory oscillations. Diabetes 1999, 48, 1557-1561. [CrossRef] [PubMed]

6. Ristow, M.; Vorgerd, M.; Mohlig, M.; Schatz, H.; Pfeiffer, A. Deficiency of phosphofructo-1-kinase/muscle subtype in humans impairs insulin secretion and causes insulin resistance. J. Clin. Investig. 1997, 100, 2833-2841. [CrossRef] [PubMed]

7. Ristow, M.; Vorgerd, M.; Mohlig, M.; Schatz, H.; Pfeiffer, A. Insulin resistance and impaired insulin secretion due to phosphofructo-1-kinase-deficiency in humans. J. Mol. Med. 1999, 77, 96-103. [CrossRef] [PubMed]

8. Da Silva, D.; Zancan, P.; Coelho, W.S.; Gomez, L.S.; Sola-Penna, M. Metformin reverses hexokinase and 6-phosphofructo-1-kinase inhibition in skeletal muscle, liver and adipose tissues from streptozotocin-induced diabetic mouse. Arch. Biochem. Biophys. 2010, 496, 53-60. [CrossRef] [PubMed]

9. Du, X.; Matsumura, T.; Edelstein, D.; Rossetti, L.; Zsengeller, Z.; Szabo, C.; Brownlee, M. Inhibition of GAPDH activity by poly(ADP-ribose) polymerase activates three major pathways of hyperglycemic damage in endothelial cells. J. Clin. Investig. 2003, 112, 1049-1057. [CrossRef] [PubMed]

10. Brownlee, M. The pathobiology of diabetic complications: A unifying mechanism. Diabetes 2005, 54, 1615-1625. [CrossRef] [PubMed]

11. Wentzel, P.; Ejdesjo, A.; Eriksson, U.J. Maternal diabetes in vivo and high glucose in vitro diminish GAPDH activity in rat embryos. Diabetes 2003, 52, 1222-1228. [CrossRef] [PubMed]

12. Senhaji, N.; Elkhalfi, B.; Soukri, A. Contribution to the study of glyceraldehyde-3-phosphate dehydrogenase in patients with type 2 diabetes. Pathol.-Biol. 2015, 63, 74-79. [CrossRef] [PubMed]

13. Befroy, D.E.; Petersen, K.F.; Dufour, S.; Mason, G.F.; de Graaf, R.A.; Rothman, D.L.; Shulman, G.I. Impaired mitochondrial substrate oxidation in muscle of insulin-resistant offspring of type 2 diabetic patients. Diabetes 2007, 56, 1376-1381. [CrossRef] [PubMed]

14. Petersen, K.F.; Dufour, S.; Befroy, D.; Garcia, R.; Shulman, G.I. Impaired mitochondrial activity in the insulin-resistant offspring of patients with type 2 diabetes. N. Engl. J. Med. 2004, 350, 664-671. [CrossRef] [PubMed]

15. Szendroedi, J.; Schmid, A.I.; Chmelik, M.; Toth, C.; Brehm, A.; Krssak, M.; Nowotny, P.; Wolzt, M.; Waldhausl, W.; Roden, M. Muscle mitochondrial ATP synthesis and glucose transport/phosphorylation in type 2 diabetes. PLoS Med. 2007, 4, e154. [CrossRef] [PubMed]

16. Patel, D.P.; Krausz, K.W.; Xie, C.; Beyoglu, D.; Gonzalez, F.J.; Idle, J.R. Metabolic profiling by gas chromatography-mass spectrometry of energy metabolism in high-fat diet-fed obese mice. PLoS ONE 2017, 12, e0177953. [CrossRef] [PubMed]

17. Patti, M.E.; Butte, A.J.; Crunkhorn, S.; Cusi, K.; Berria, R.; Kashyap, S.; Miyazaki, Y.; Kohane, I.; Costello, M.; Saccone, R.; et al. Coordinated reduction of genes of oxidative metabolism in humans with insulin resistance and diabetes: Potential role of PGC1 and NRF1. Proc. Natl. Acad. Sci. USA 2003, 100, 8466-8471. [CrossRef] [PubMed]

18. Ritov, V.B.; Menshikova, E.V.; He, J.; Ferrell, R.E.; Goodpaster, B.H.; Kelley, D.E. Deficiency of subsarcolemmal mitochondria in obesity and type 2 diabetes. Diabetes 2005, 54, 8-14. [CrossRef] [PubMed]

19. Apontes, P.; Liu, Z.; Su, K.; Benard, O.; Youn, D.Y.; Li, X.; Li, W.; Mirza, R.H.; Bastie, C.C.; Jelicks, L.A.; et al. Mangiferin Stimulates Carbohydrate Oxidation and Protects against High Fat Diet Induced Metabolic Disorders. Diabetes 2014, 63, 3626-3636. [CrossRef] [PubMed]

20. Kirby, D.M.; Thorburn, D.R.; Turnbull, D.M.; Taylor, R.W. Biochemical assays of respiratory chain complex activity. Methods Cell Biol. 2007, 80, 93-119. [PubMed]

21. Puigserver, P. Tissue-specific regulation of metabolic pathways through the transcriptional coactivator PGC1- $\alpha$. Int. J. Obes. 2005, 29, S5-S9. [CrossRef] [PubMed]

22. Vega, R.B.; Huss, J.M.; Kelly, D.P. The coactivator PGC-1 cooperates with peroxisome proliferator-activated receptor $\alpha$ in transcriptional control of nuclear genes encoding mitochondrial fatty acid oxidation enzymes. Mol. Cell Biol. 2000, 20, 1868-1876. [CrossRef] [PubMed]

23. Huss, J.M.; Kopp, R.P.; Kelly, D.P. Peroxisome proliferator-activated receptor coactivator- $1 \alpha(\mathrm{PGC}-1 \alpha)$ coactivates the cardiac-enriched nuclear receptors estrogen-related receptor-alpha and -gamma. Identification of novel leucine-rich interaction motif within PGC-1 $\alpha$. J. Biol. Chem. 2002, 277, 40265-40274. [CrossRef] [PubMed] 
24. Wu, Y.; Delerive, P.; Chin, W.W.; Burris, T.P. Requirement of helix 1 and the AF-2 domain of the thyroid hormone receptor for coactivation by PGC-1. J. Biol. Chem. 2002, 277, 8898-8905. [CrossRef] [PubMed]

25. Scarpulla, R.C. Nuclear control of respiratory chain expression by nuclear respiratory factors and PGC-1-related coactivator. Ann. N. Y. Acad. Sci. 2008, 1147, 321-334. [CrossRef] [PubMed]

26. Rodgers, J.T.; Lerin, C.; Haas, W.; Gygi, S.P.; Spiegelman, B.M.; Puigserver, P. Nutrient control of glucose homeostasis through a complex of PGC-1 $\alpha$ and SIRT1. Nature 2005, 434, 113-118. [CrossRef] [PubMed]

27. Sellamuthu, P.S.; Muniappan, B.P.; Perumal, S.M.; Kandasamy, M. Antihyperglycemic Effect of Mangiferin in Streptozotocin Induced Diabetic Rats. J. Health Sci. 2009, 55, 206-214. [CrossRef]

28. Simi, B.; Sempore, B.; Mayet, M.H.; Favier, R.J. Additive effects of training and high-fat diet on energy metabolism during exercise. J. Appl. Physiol. (1985) 1991, 71, 197-203. [CrossRef] [PubMed]

29. Leibowitz, S.F.; Dourmashkin, J.T.; Chang, G.Q.; Hill, J.O.; Gayles, E.C.; Fried, S.K.; Wang, J. Acute high-fat diet paradigms link galanin to triglycerides and their transport and metabolism in muscle. Brain Res. 2004, 1008, 168-178. [CrossRef] [PubMed]

30. Roy, C.; Paglialunga, S.; Fisette, A.; Schrauwen, P.; Moonen-Kornips, E.; St-Onge, J.; Hesselink, M.K.; Richard, D.; Joanisse, D.R.; Cianflone, K. Shift in metabolic fuel in acylation-stimulating protein-deficient mice following a high-fat diet. Am. J. Physiol. Endocrinol. Metab. 2008, 294, E1051-E1059. [CrossRef] [PubMed]

31. Chen-Zion, M.; Livnat, T.; Beitner, R. Effects of long-term streptozotocin diabetes on cytoskeletal and cytosolic phosphofructokinase and the levels of glucose 1,6-bisphosphate and fructose 2,6-bisphosphate in different rat muscles. Biochem. Med. Metab. Biol. 1994, 53, 137-144. [CrossRef] [PubMed]

32. Vestergaard, H. Studies of gene expression and activity of hexokinase, phosphofructokinase and glycogen synthase in human skeletal muscle in states of altered insulin-stimulated glucose metabolism. Dan. Med. Bull. 1999, 46, 13-34. [PubMed]

33. Aithal, H.N.; Walsh-Reitz, M.M.; Toback, F.G. Regulation of glyceraldehyde-3-phosphate dehydrogenase by a cytosolic protein. Am. J. Physiol. 1985, 249, C111-C116. [CrossRef] [PubMed]

34. Nicholls, C.; Li, H.; Liu, J.P. GAPDH: A common enzyme with uncommon functions. Clin. Exp. Pharmacol. Physiol. 2012, 39, 674-679. [CrossRef] [PubMed]

35. Modrego, J.; de las Heras, N.; Zamorano-Leon, J.J.; Mateos-Caceres, P.J.; Martin-Fernandez, B.; Valero-Munoz, M.; Lahera, V.; Lopez-Farre, A.J. Changes in cardiac energy metabolic pathways in overweighed rats fed a high-fat diet. Eur. J. Nutr. 2013, 52, 847-856. [CrossRef] [PubMed]

36. Sellayah, D.; Sek, K.; Anthony, F.W.; Hanson, M.A.; Cagampang, F.R. Sensitivity of housekeeping genes in the hypothalamus to mismatch in diets between pre- and postnatal periods in mice. Neurosci. Lett. 2008, 447, 54-57. [CrossRef] [PubMed]

37. Xu, L.; Ma, X.; Cui, B.; Li, X.; Ning, G.; Wang, S. Selection of reference genes for qRT-PCR in high fat diet-induced hepatic steatosis mice model. Mol. Biotechnol. 2011, 48, 255-262. [CrossRef] [PubMed]

38. Van Vranken, J.G.; Na, U.; Winge, D.R.; Rutter, J. Protein-mediated assembly of succinate dehydrogenase and its cofactors. Crit. Rev. Biochem. Mol. Biol. 2015, 50, 168-180. [CrossRef] [PubMed]

39. Bourgeron, T.; Rustin, P.; Chretien, D.; Birch-Machin, M.; Bourgeois, M.; Viegas-Pequignot, E.; Munnich, A.; Rotig, A. Mutation of a nuclear succinate dehydrogenase gene results in mitochondrial respiratory chain deficiency. Nat. Genet. 1995, 11, 144-149. [CrossRef] [PubMed]

40. Acevedo, L.M.; Raya, A.I.; Martinez-Moreno, J.M.; Aguilera-Tejero, E.; Rivero, J.L. Mangiferin protects against adverse skeletal muscle changes and enhances muscle oxidative capacity in obese rats. PLoS ONE 2017, 12, e0173028. [CrossRef] [PubMed]

41. Prabhu, S.; Jainu, M.; Sabitha, K.E.; Shyamala Devi, C.S. Effect of mangiferin on mitochondrial energy production in experimentally induced myocardial infarcted rats. Vasc. Pharmacol. 2006, 44, 519-525. [CrossRef] [PubMed]

42. Lim, J.; Liu, Z.; Apontes, P.; Feng, D.; Pessin, J.E.; Sauve, A.A.; Angeletti, R.H.; Chi, Y. Dual mode action of mangiferin in mouse liver under high fat diet. PLoS ONE 2014, 9, e90137. [CrossRef] [PubMed]

43. Muruganandan, S.; Srinivasan, K.; Gupta, S.; Gupta, P.K.; Lal, J. Effect of mangiferin on hyperglycemia and atherogenicity in streptozotocin diabetic rats. J. Ethnopharmacol. 2005, 97, 497-501. [CrossRef] [PubMed]

44. Guo, F.; Huang, C.; Liao, X.; Wang, Y.; He, Y.; Feng, R.; Li, Y.; Sun, C. Beneficial effects of mangiferin on hyperlipidemia in high-fat-fed hamsters. Mol. Nutr. Food Res. 2011, 55, 1809-1818. [CrossRef] [PubMed] 
45. Niu, Y.; Li, S.; Na, L.; Feng, R.; Liu, L.; Li, Y.; Sun, C. Mangiferin decreases plasma free fatty acids through promoting its catabolism in liver by activation of AMPK. PLoS ONE 2012, 7, e30782. [CrossRef] [PubMed]

46. Xing, X.; Li, D.; Chen, D.; Zhou, L.; Chonan, R.; Yamahara, J.; Wang, J.; Li, Y. Mangiferin treatment inhibits hepatic expression of acyl-coenzyme A:diacylglycerol acyltransferase- 2 in fructose-fed spontaneously hypertensive rats: A link to amelioration of fatty liver. Toxicol. Appl. Pharmacol. 2014, 280, 207-215. [CrossRef] [PubMed]

47. Zhou, L.; Pan, Y.; Chonan, R.; Batey, R.; Rong, X.; Yamahara, J.; Wang, J.; Li, Y. Mitigation of Insulin Resistance by Mangiferin in a Rat Model of Fructose-Induced Metabolic Syndrome Is Associated with Modulation of CD36 Redistribution in the Skeletal Muscle. J. Pharmacol. Exp. Ther. 2016, 356, 74-84. [CrossRef] [PubMed]

48. Na, L.X.; Zhang, Q.; Jiang, S.; Du, S.S.; Zhang, W.; Li, Y.; Sun, C.H.; Niu, Y.C. Mangiferin supplementation improves serum lipid profiles in overweight patients with hyperlipidemia: A double-blind randomized controlled trial. Sci. Rep.-UK 2015, 5. [CrossRef] [PubMed]

49. Han, D.; Chen, C.; Zhang, C.; Zhang, Y.; Tang, X. Determination of mangiferin in rat plasma by liquid-liquid extraction with UPLC-MS/MS. J. Pharm. Biomed. Anal. 2010, 51, 260-263. [CrossRef] [PubMed]

50. Hou, S.Y.; Wang, F.; Li, Y.M.; Li, Y.; Wang, M.Q.; Sun, D.J.; Sun, C.H. Pharmacokinetic study of mangiferin in human plasma after oral administration. Food Chem. 2012, 132, 289-294. [CrossRef] [PubMed]

51. Ma, H.; Chen, H.; Sun, L.; Tong, L.; Zhang, T. Improving permeability and oral absorption of mangiferin by phospholipid complexation. Fitoterapia 2014, 93, 54-61. [CrossRef] [PubMed]

52. Serasinghe, M.N.; Wieder, S.Y.; Renault, T.T.; Elkholi, R.; Asciolla, J.J.; Yao, J.L.; Jabado, O.; Hoehn, K.; Kageyama, Y.; Sesaki, H.; et al. Mitochondrial Division Is Requisite to RAS-Induced Transformation and Targeted by Oncogenic MAPK Pathway Inhibitors. Mol. Cell 2015, 57, 521-536. [CrossRef] [PubMed]

53. Jeoung, N.H.; Sanghani, P.C.; Zhai, L.; Harris, R.A. Assay of the pyruvate dehydrogenase complex by coupling with recombinant chicken liver arylamine $N$-acetyltransferase. Anal. Biochem. 2006, 356, 44-50. [CrossRef] [PubMed]

54. Constantin-Teodosiu, D.; Cederblad, G.; Hultman, E. A sensitive radioisotopic assay of pyruvate dehydrogenase complex in human muscle tissue. Anal. Biochem. 1991, 198, 347-351. [CrossRef]

55. Drew, B.; Phaneuf, S.; Dirks, A.; Selman, C.; Gredilla, R.; Lezza, A.; Barja, G.; Leeuwenburgh, C. Effects of aging and caloric restriction on mitochondrial energy production in gastrocnemius muscle and heart. Am. J. Physiol. Regul. Integr. Comp. Physiol. 2003, 284, R474-R480. [CrossRef] [PubMed]

56. Ajaz, S.; Czajka, A.; Malik, A. Accurate measurement of circulating mitochondrial DNA content from human blood samples using real-time quantitative PCR. Methods Mol. Biol. 2015, 1264, 117-131. [PubMed] 